\title{
EVALUATING DRIVERS'STATES IN SLEEPINESS COUNTERMEASURES EXPERIMENTS USING PHYSIOLOGICAL AND EYE DATA - HYBRID LOGISTIC AND LINEAR REGRESSION MODEL
}

\author{
Elisabeth Schmidt ${ }^{1,2}$, Judith Ochs ${ }^{1}$, Ralf Decke ${ }^{1}$, Angelika C. Bullinger ${ }^{2}$ \\ ${ }^{1} \mathrm{BMW}$ AG, Munich, Germany \\ ${ }^{2}$ Technical University Chemnitz, Chemnitz, Germany \\ Email: Elisabeth.Schmidt@bmw.de
}

\begin{abstract}
Summary: Objective sleepiness evaluation is essential for the effect analysis of countermeasures for driver sleepiness, such as in-car stimulants. Furthermore, measuring drivers' sleepiness in simulator studies also becomes important when investigating causes for task-related sleepiness, for example driving on monotonous routes, which requires little driver engagement. To evaluate driver sleepiness and the effect of countermeasures, we developed a model for predicting sleepiness using both simple logistic and linear regression of heart rate variability, skin conductance and pupil diameter. The algorithm was trained and tested with data from 88 participants in driving simulator studies. A prediction accuracy of $77 \%$ was achieved and the model's sensitivity to thermal stimulation was shown.
\end{abstract}

\section{INTRODUCTION}

In the last decades, driver fatigue theories (Lal \& Craig, 2001; May \& Baldwin, 2009; van Veen et al., 2014) have been developed that distinguish different types of fatigue. Most theories differentiate task-related (TR) and sleep-related (SR) fatigue. TR fatigue can be due to task overload or underload. The latter one is also referred to as cognitive sleepiness. This differentiation becomes important when considering countermeasures for driver fatigue, as the literature reviews of May \& Baldwin (2009) and van Veen et al. (2014) propose various treatments for different fatigue types. In the case of TR fatigue due to monotony, countermeasures include a variety of in-car stimulations. To research driving comfort, studies have been conducted investigating these countermeasures (Desmond \& Matthews, 1997; van Veen, 2016).

A tool for measuring sleepiness when investigating driver fatigue and the effect of different stimuli in experimental settings, is subjective questionnaires (e.g. Karolinska Sleepiness Scale, KSS). Questionnaires can only be asked, however, at discrete times, preventing a continuous analysis of sleepiness. Furthermore, listening and answering questionnaires has an awakening effect on the driver, which is often undesirable in driver sleepiness research.

A different tool for measuring sleepiness is classification algorithms. Patel et al. (2011) for example describe a neural network classifying sleepiness with $90 \%$ accuracy based on drivers' ECG (electrocardiogram) data. Friedrichs \& Yang (2010) and Hu \& Zheng (2009) report accuracies of $83 \%$ when differentiating three degrees of sleepiness based on eye data. These algorithms have been developed using data from sleep-deprived drivers, hence these are detecting SR fatigue. Another model, from Igasaki et al. (2015), uses data of non-sleep-deprived drivers. Their logistic regression based on heart rate variability (HRV) measures and respiratory features yields $81 \%$ detection accuracy, however, it was only generated with data from eight 
male drivers. None of the above studies attempted to model sensitivity to in-car sleepiness countermeasures.

We propose an algorithm that can be used as a tool for continuously evaluating driver sleepiness in simulator studies with a focus on TR sleepiness caused by monotonous driving. The training data were generated by means of a secondary data analysis of three driving simulator experiments investigating in-car countermeasures on the driver's state. Our leading research questions are:

RQ1: How accurately can the algorithm detect cognitive sleepiness in an unknown driver? RQ2: Can the algorithm detect changes in sleepiness induced by countermeasures?

To answer these questions, this paper first describes how training data were collected from three simulator studies and the selection of features indicating sleepiness. Second, the algorithm, consisting of a cascaded logistic and linear regression model, is detailed with its quality factors. Finally, our research questions are discussed and the applicational limitations are reflected on.

\section{METHOD}

\section{Experimental Designs}

A series of driving simulator experiments provided the data for the training and testing of the sleepiness classification. The primary aim of these pilot experiments was the investigation of countermeasures for critical driver states. The purpose of this secondary data analysis is the modeling of sleepiness caused by task underload. Table 1 shows a description of the monotonous highway drives, information on sample sizes and a description of the treatment.

The first experiment included a 24 min. long monotonous drive, visualized in Table 1 . After 14 min. and at the end of the drive, participants answered the Stanford Sleepiness Scale (SSS). A countermeasure was applied between min. 20 and 23 that consisted of a combination of orange light from the car ceiling, scent, rhythmic sound and an increased fan intensity of the AC (COMB). The investigator started these in-car settings via remote control. In our second study (Schmidt et al., 2017), each subject drove two identical highway routes for $26 \mathrm{~min}$. In one of the drives, cooling at $17^{\circ} \mathrm{C}(\mathrm{COOL})$ was applied between min. 20 and 26. Subjects evaluated their sleepiness after 6, 16 and $26 \mathrm{~min}$. with the KSS. The third experiment included an $18 \mathrm{~min}$. long drive with no intervention (CONTROL). At the end of the drive, the participants ranked their subjective sleepiness with the SSS.

All drives were highway drives with very little traffic. Based on the observations of Schmidt et al. (2016) who investigated the possibility to induce cognitive sleepiness by means of traffic scenarios, it was concluded that $17 \mathrm{~min}$. of monotonous driving in a simulator is sufficient to evoke high SSS ratings. The test vehicle was a street-legal car, placed in a static driving simulator with a curved screen providing a $220^{\circ}$ view. Two monitors facing the side mirrors placed behind the car and a rear mirror display simulated a rear view. For each of the three experiments, 50 subjects were invited. All 150 subjects maintained their regular sleep schedule. Of those, $n=28$ datasets were excluded from analysis due to sensor failure or simulator sickness. ECG and skin conductance level (SCL) were measured with medical sensors (g.tech, Austria) 
with a sampling frequency of $512 \mathrm{~Hz}$. Gaze coordinates and pupil diameter of each eye were recorded at $60 \mathrm{~Hz}$ using a remote eye tracker (Tobii, Sweden).

Table 1. Overview of simulator drives used for algorithm development

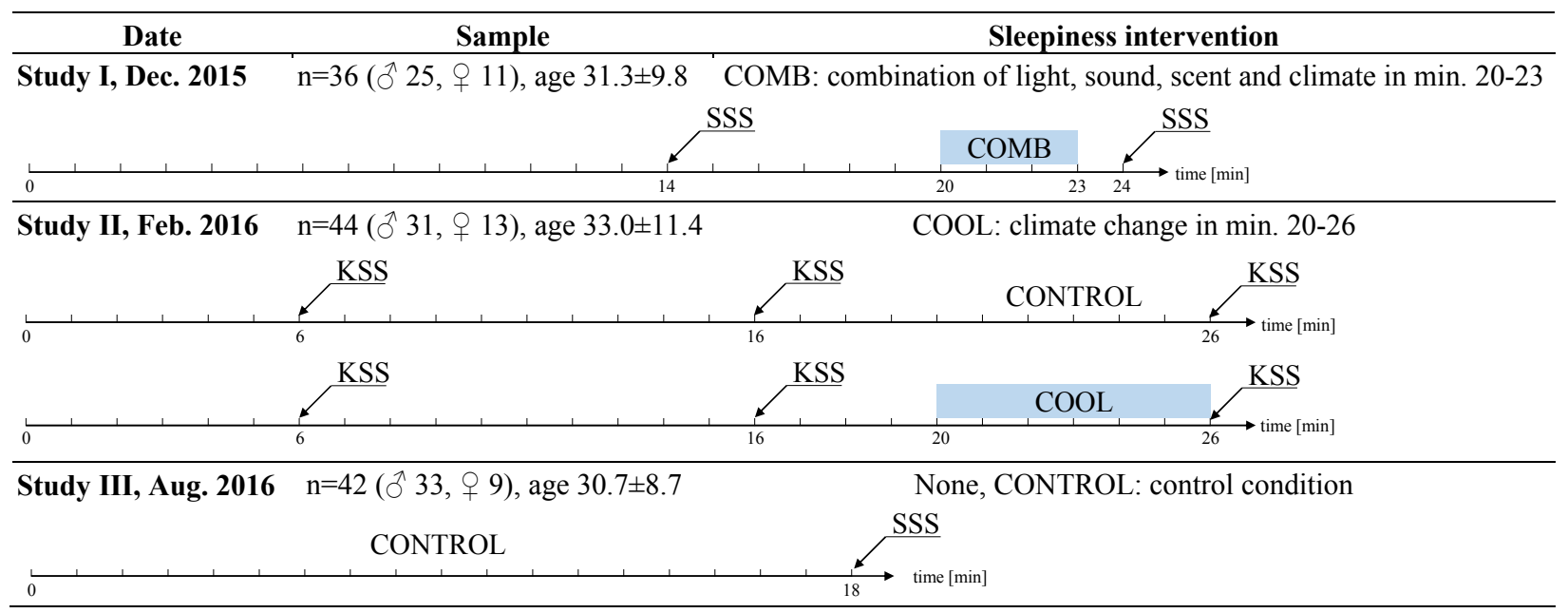

\section{Signal Processing}

Predictor variables. The data were processed and analyzed using Matlab 2013b and the algorithm was developed with Weka 3.6.13 (Hall et al., 2009). Figure 1 visualizes the different processing steps. The time domain HRV measures SDNN (standard deviation of normal to normal intervals) and RMSSD (root mean square of successive differences) for the period of 3 min. were extracted from the ECG recordings. Furthermore, a spectral analyses of a moving 3min. sequence of interbeat intervals was performed and the frequency domain HRV measures LF (low frequency component, 0.04-0.15 Hz), HF (high frequency component, 0.15-0.4 Hz) and total power were obtained. Pupil diameters of both eyes were averaged for each subject.

To increase the classification accuracies, the features were further transformed. Instead of using absolute values, we found that the relative changes of features compared to the first driving minute (third minute respectively for HRV measures) yields better results. The subscript 'rel' in equations 1, 2 and 3 indicates the relative change of the parameters over time compared to their respective value at the beginning of the measurement. Furthermore, the exponents of SDNN,

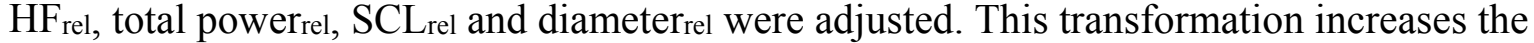
kurtosis in their respective distributions up to 9 times and reduces the overall variability of the parameters.

$$
\begin{gathered}
\text { diameter }_{\text {rel }}(t)=\frac{\text { diameter }(t)-\operatorname{diameter}(1)}{\text { diameter }(1)}, t \ldots \text { time }[\mathrm{min}] \\
S C L_{r e l}(t)=\frac{S C L(t)-S C L(1)}{S C L(1)}, \quad H R V_{\text {rel }}(t)=\frac{H R V(t)-H R V(3)}{H R V(3)}
\end{gathered}
$$

Labels. The responses of the subjects in the KSS and SSS were used for labeling the data. The corresponding predictor variables are the values of the features in the min. before the subjective rating (e.g. the KSS rating after $16 \mathrm{~min}$. was matched with the features from the $16^{\text {th }} \mathrm{min}$.). The classes "awake" and "sleepy" were formed by means of the subjective sleepiness rankings in the 
following way: data observations with KSS-values of 1, 2, 3 and 4 as well as SSS-values of 1, 2 and 3 formed class 1 - "awake". Data observations with KSS-values of 8 and 9 as well as SSSvalues of 6 and 7 formed class 2 - "sleepy". This way, a total data set of 171 observations from 88 different drivers was generated. The dataset includes 85 observations of awake drivers and 86 observations of tired drivers. An amount of 207 observations with mid-range KSS and SSS values was removed from the training and testing.

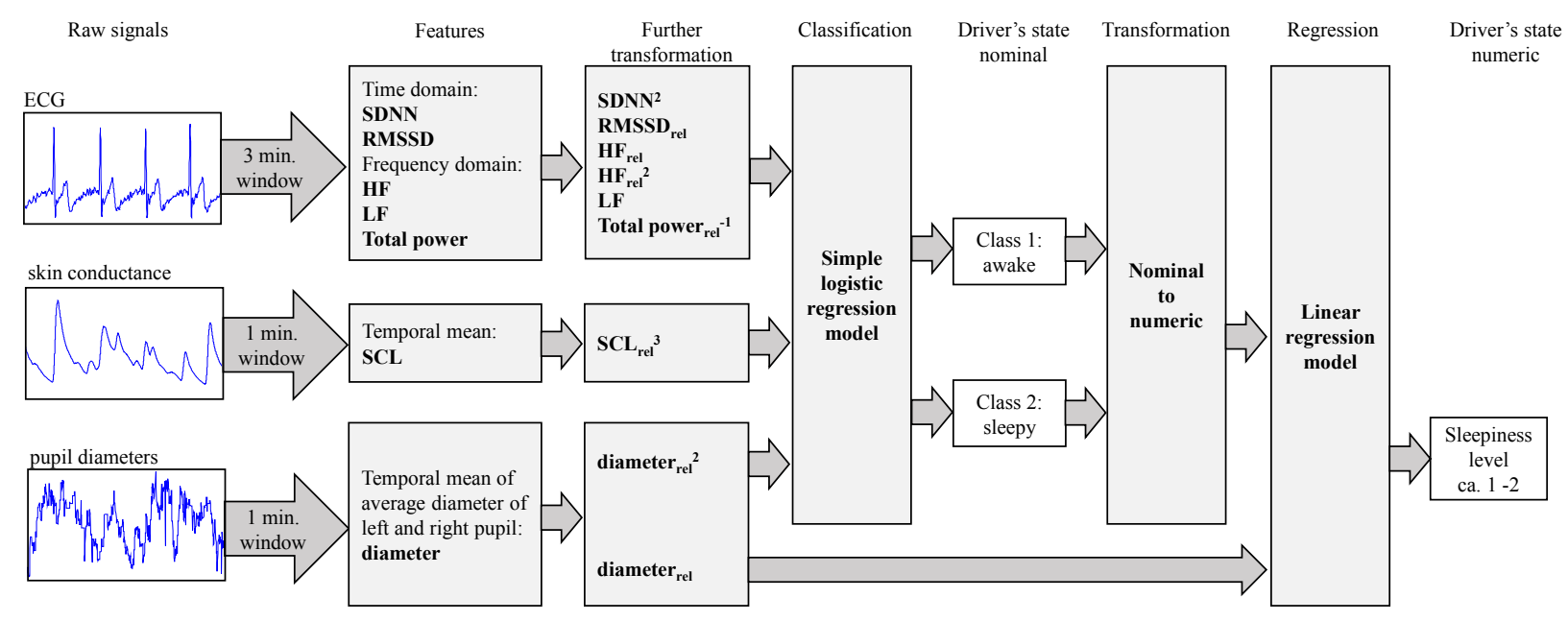

\section{Algorithm}

Figure 1. Signal processing

The detection of sleepiness can be handled in two different ways: classification or regression. This is possible because the classes 1 and 2 do not only describe nominal classes (awake and sleepy) but can also serve as numeric values for the degree of sleepiness, allowing for regression approaches. Regression approaches often fail to model the individual differences in prediction problems because the prediction is often approximating the mean of all labels. Therefore, we chose a classification approach over regression in the first step to distinguish the separate classes. To improve the sensitivity of the prediction results to external stimuli, a linear regression model with the inputs diameter rel and the class values was developed in the second step.

\section{RESULTS}

After comparing several classification algorithms, we found that the logistic regression classifier performed the best in terms of classification accuracy. The logistic regression model was developed using a 10-fold-cross-validation on the 171 observations. The logistic regression function is given by equations (4) and (5) with a coefficient vector (a, b, c, d, e, f, g, h, i) of $\left(8.4 \cdot 10^{-5}, 0.81,-0.84,0.16,-6.5 \cdot 10^{-7}, 4.1 \cdot 10^{-3}, 4.6,-6.6,0.45\right)$. The classification accuracy is $77.19 \%$, with a ROC area of 0.781 for both classes. Figure 2 shows that a total of 92 observations were classified as awake, 79 as sleepy. The confusion matrix is shown in Table 2 .

$$
\begin{gathered}
\text { probabilty of class } 1=\frac{1}{1+\exp (-x)} \\
x=\mathrm{a} \cdot S D N N^{2}+\mathrm{b} \cdot R M S S D_{r e l}+\mathrm{c} \cdot H F_{r e l}+\mathrm{d} \cdot H F_{r e l}^{2}+\mathrm{e} \cdot L F \\
+\mathrm{f} \cdot \text { total Power } \\
\text { probability of class } 2=\mathrm{g} \cdot G S R_{r e l}^{3}+\mathrm{h} \cdot \text { diameter }_{r e l}^{2}+\mathrm{i} \\
\text { probability of class } 1
\end{gathered}
$$


Even though the classification of cognitive sleepiness is fair, it is not sufficiently accurate to model the activation of the driver through the countermeasures because the class is either " 1 awake" or "2-sleepy". Hence, the model does not represent any intermediate state, such as slight reductions in sleepiness due to in-car stimulation.

Table 2. Confusion matrix of simple logistic

\begin{tabular}{ccc}
\multicolumn{3}{c}{ regression } \\
\hline Classified as $\rightarrow$ & $\mathbf{1}$ & $\mathbf{2}$ \\
\hline $\mathbf{1}$ & 69 & 16 \\
\hline $\mathbf{2}$ & 23 & 63 \\
\hline
\end{tabular}

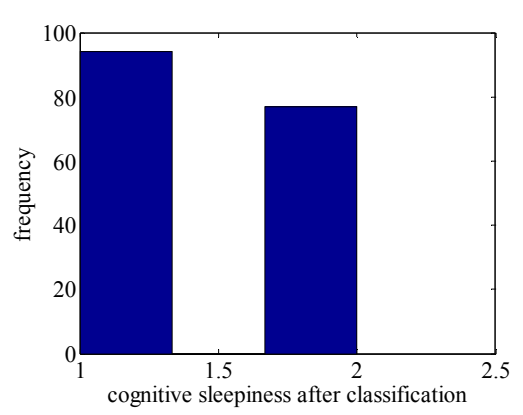

Figure 2. Histogram of predicted sleepiness with simple logistic regression
Table 3. Confusion matrix of hybrid logistic and linear

\begin{tabular}{ccc}
\multicolumn{2}{c}{ regression } \\
\hline Classified as $\rightarrow$ & $\mathbf{1}$ & $\mathbf{2}$ \\
\hline $\mathbf{1}$ & 70 & 15 \\
\hline $\mathbf{2}$ & 24 & 62
\end{tabular}

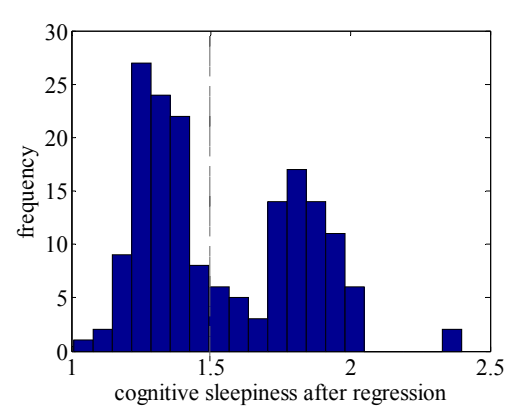

Figure 3. Histogram of predicted sleepiness with hybrid logistic and linear regression

Therefore, the model was improved by cascading a linear regression model after the logistic classifier, which reproduces such intermediate states. The classification result serves as input for the linear regression model, along with the relative change in pupil diameter. The pupil diameter was chosen as an input because it is a very sensitive measure of sympathetic activation and hence replicating slight changes of driver activation. The linear regression model for sleepiness was generated using a 10-fold-cross-validation. The regression function is given by equation (7) with a coefficient vector $(\mathrm{j}, \mathrm{k}, 1)$ of $(0.45,-1.5,0.8)$. The correlation coefficient of the regression model is $\mathrm{r}=0.53, \mathrm{p}<.001$. The classification accuracy of the rounded numeric sleepiness level is still $77.19 \%$. Table 3 shows the new confusion matrix and Figure 3 the new distribution of predicted sleepiness levels.

$$
\text { sleepiness level }=\mathrm{j} \cdot \text { class }+\mathrm{k} \cdot \text { diameter }_{\text {rel }}+\mathrm{l}
$$

A comparison of the distributions in Figure 2 and Figure 3 visualizes the effect of cascading the linear regression: The linear regression approximates the mean of all observations, which means that the two bars of Figure 2 move closer towards the mean of 1.5 in Figure 3. This hardly alters the classification accuracy (see Table 2 and Table 3), but allows for the detection of slight changes in sleepiness induced by external stimuli. If the linear regression would have been performed without the logistic classifier beforehand, the distribution of predictions would peak at 1.5 and therefore, confuse sleepy and awake drivers.

To evaluate the sensitivity of the proposed algorithms to changes in cognitive sleepiness due to stimulation, we compared the predicted sleepiness of the two drives "CONTROL" and "COOL" of the simulator study II. Figure 4 and 5 show the mean sleepiness of the 44 drivers for the logistic and the hybrid logistic and linear regression respectively and it can be seen that sleepiness increased over the course of both drives. The logistic regression model (Figure 4) fails to model the slight reduction in sleepiness due to the cooling treatment because there are hardly significant differences between the "CONTROL" and "COOL" condition in min. 20 to 26. For 
the hybrid regression approach (Figure 5), the t-test results for each driving min. yield statistically significant differences between the two conditions in the $21^{\text {st }}(p<0.003), 23^{\text {rd }}$ $(p<0.049), 24^{\text {th }}(p<0.034), 25^{\text {th }}(p<0.023)$ and $26^{\text {th }}(p<0.001)$ min., in which cooling was applied. The graph also shows that there is a trend for decreased sleepiness after 6 and 16 min., when the drivers responded to the KSS, which had an awakening effect.

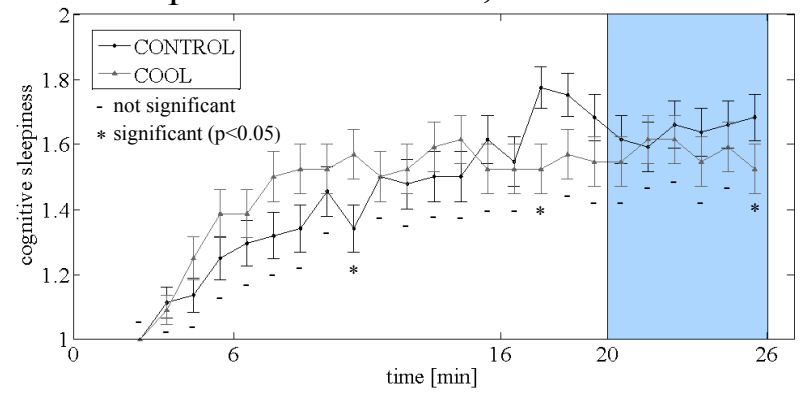

Figure 4. Mean and standard error of predicted sleepiness through logistic regression with t-test results between conditions

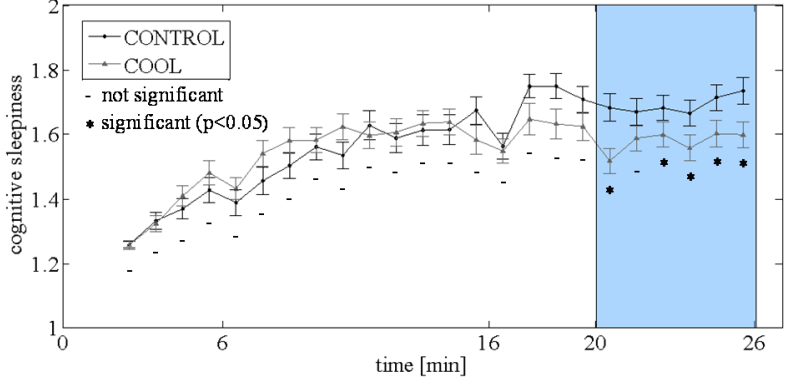

Figure 5. Mean and standard error of predicted sleepiness through hybrid logistic and linear regression with t-test results between conditions

\section{CONCLUSION}

Based on the presented results, the research questions can be answered:

A1: The cognitive sleepiness of car simulator drivers can be detected with an accuracy of 77.19\% using ECG, SCL and pupil diameter as inputs. The proposed algorithm achieves a fair classification accuracy, taking into account that the data were collected from non-sleep-deprived drivers. Better performing algorithms with similar signal input requirements found in the literature were trained with data from sleep-deprived drivers. It is questionable, however, whether these algorithms can also predict TR sleepiness as accurately.

A2: The algorithm detects changes in sleepiness of the sample in our second study which were induced by thermal stimulation. Furthermore, the predicted values also reflect the awakening effect when the driver is answering the KSS. Though, when applying the model for the evaluation of sleepiness countermeasures, we recommend having a large sample to overcome the imprecision of the prediction.

When comparing the classification results of the logistic regression with the results of the hybrid logistic and linear regression, there is no improvement in classification accuracy by cascading the linear regression in the hybrid model. This additional step, however, reproduces slight changes in sleepiness by allowing for continuous sleepiness values. Furthermore, this linear regression is strongly influenced by the relative change in pupil diameter, which is a very sensitive measure of activation.

The proposed algorithm will perform well only if the light settings are kept constant during the experiment. The reason is that changes in the brightness cause pupillary restrictions unrelated to an increase in sleepiness and would therefore skew both classification and regression results. For this reason, the algorithm is not suited to evaluate light as an intervening stimulant for sleepy drivers. Since we trained the algorithm with the extreme KSS- and SSS-values, the reported accuracy can only be guaranteed for awake or sleepy ratings. For intermediate values $(5,6,7$ for KSS and 4, 5 for SSS) the logistic regression probabilities are close to an equal likelihood for both classes, increasing the risk of misclassifications. 
Taking both the accuracy and sensitivity of the regression model into account, the algorithm is a suitable tool for continuously evaluating TR sleepiness due to monotony in driving simulator studies. The model can also serve as an objective measure for the effectiveness of countermeasures, such as in-car stimulants. In further studies, we would like to evaluate the performance of the sleepiness prediction for repeated and even cooler thermal stimuli. Moreover, the algorithm should also be tested for different causes of TR sleepiness, e.g. automated driving.

\section{REFERENCES}

Desmond, P. A., \& Matthews, G. (1997). Implications of task-induced fatigue effects for invehicle countermeasures to driver fatigue. Accident Analysis \& Prevention, 29(4), 515-523.

Friedrichs, F., \& Yang, B. (2010). Camera-based drowsiness reference for driver state classification under real driving conditions. Proceedings of the IEEE Intelligent Vehicles Symposium, San Diego, California, 101-106.

Hall, M., Frank, E., Holmes, G., Pfahringer, B., Reutemann, P., \& Witten, I. (2009). The WEKA data mining software: an update. ACM SIGKDD explorations newsletter, 11(1), 10-18.

Hu, S., \& Zheng, G. (2009). Driver drowsiness detection with eyelid related parameters by Support Vector Machine. Expert Systems with Applications, 36(4), 7651-7658.

Igasaki, T., Nagasawa, K., Murayama, N., \& Hu, Z. (2015). Drowsiness estimation under driving environment by heart rate variability and/or breathing rate variability with logistic regression analysis. Proceedings of the 8th IEEE International Conference on Biomedical Engineering and Informatics, Shenyang, China, 189-193.

Lal, S. K., \& Craig, A. (2001). A critical review of the psychophysiology of driver fatigue. Biological psychology, 55(3), 173-194.

May, J. F., \& Baldwin, C. L. (2009). Driver fatigue: The importance of identifying causal factors of fatigue when considering detection and countermeasure technologies. Transportation Research Part F: Traffic Psychology and Behaviour, 12(3), 218-224.

Patel, M., Lal, S. K. L., Kavanagh, D., \& Rossiter, P. (2011). Applying neural network analysis on heart rate variability data to assess driver fatigue. Expert systems with Applications, 38(6), 7235-7242.

Schmidt, E., Decke, R., \& Rasshofer, R. (2016). Correlation between subjective driver state measures and psychophysiological and vehicular data in simulated driving. Proceedings of the IEEE Intelligent Vehicles Symposium, Gothenburg, Sweden, 1380-1385.

Schmidt, E., Decke, R., Rasshofer, R., \& Bullinger, A. C. (2017). Psychophysiological responses to short-term cooling during a simulated monotonous driving task. Applied Ergonomics, 62, 9-18.

Van Veen, S., Vink, P., Franz, M., \& Wagner, P.-O. (2014). Enhancing the vigilance of car drivers: a review on fatigue caused by the driving task and possible countermeasures. Proceedings of the 5th International Conference on Applied Human Factors and Ergonomics, Kraków, Poland, 516-525.

Van Veen, S. (2016). Driver Vitalization - Investigating sensory stimulation to achieve a positive driving experience. $\mathrm{PhD}$ thesis, TU Delft. 